\title{
Stochastic behaviour of mistuned stator vane sectors: An industrial application
}

\author{
A.C. Sall ${ }^{\mathrm{a}, \mathrm{b}}$, F. Thouverez ${ }^{\mathrm{a}}$, L. Blanc ${ }^{\mathrm{a}, *}$ and P. Jean ${ }^{\mathrm{b}}$ \\ ${ }^{\mathrm{a}}$ École Centrale de Lyon, Laboratoire de Tribologie et Dynamique des Systèmes, Écully Cedex, France \\ ${ }^{\mathrm{b}}$ Snecma, Safran group, Moissy-Cramayel, France
}

\begin{abstract}
Stator vanes which are found in axial compressors are subject to vibratory fatigue. Their division into monoblock sectors makes the prediction of their vibratory behaviour difficult by deterministic methods due to the loss of the cyclic symmetry properties and also to a high sensitivity to mistuning. The purpose is to present a robust calculation strategy based on a stochastic modelisation of the structure. The methodology has been developed first on a simplified model and then applied to an industrial case. Polynomial chaos based results are in good agreement with reference Monte Carlo simulations.
\end{abstract}

Keywords: Blades vibration, mistuning, uncertainties, polynomial chaos

\section{Introduction}

The fatigue design of aircraft engines components such as bladed disks requires necessarily their dynamic analysis. Assuming cyclic symmetry properties, the dynamic study of a single blade sector is sufficient to know the behaviour of the whole disk by decomposing the response into Fourier series $[12,13,15]$. This technique provides an interesting computation time but also a better precision of the results because it is possible to use very fine meshes. However, the geometric and material dispersion due to the manufacturing tolerances, sources of mistuning, makes the assumption of cyclic symmetry far from reality. Indeed, the mistuning has a non-negligible effect on the vibratory behaviour of the bladed disks by localizing an important part of the energy on a reduced number of blades which can lead to a premature vibratory fatigue of the component $[2,5,11,14]$. This phenomenon of modal localization is conditioned, on the one hand, by the coupling stiffness between the blades and the disk and, on the other hand, by the rate of mistuning.

In this study, the parts of interest are the stator vanes of aircraft engines. Their role is to straighten the air flow from the rotors located upstream to increase the static pressure. The new technology consisting in dividing into sectors those stator vanes leads to consider independent stator vane sectors with different numbers of blades. So, the cyclic symmetry conditions cannot be employed anymore even if mistuning is taken into account. The vibratory behaviour of a tuned stator vane sector is characterized by a high modal density in some frequency ranges, which corresponds to the blade flexural and torsion modes. This phenomenon results in a high sensitivity of these modes to the mistuning within the stator vane. The purpose of this work is to develop a strategy allowing to predict the vibratory behaviour of monoblock sectored stator vanes in a robust way. Indeed, by determining the most mistuning sensitive areas of a given sector - in terms of local vibratory amplitude probability - the engineering departments will be able to optimize the design of the sector or the strain gauges positioning on prototype stator vanes.

In the first part of this paper, general results related to the vibratory behaviour of stator vane sectors and to their sensitivity to mistuning are presented. The details of these results can be found in [10]. The second part deals with

\footnotetext{
*Corresponding author: L. Blanc, École Centrale de Lyon, Laboratoire de Tribologie et Dynamique des Systèmes, Équipe Dynamique des Structures et des Systèmes, 36 avenue Guy de Collongue, 69134 Écully Cedex, France. E-mail: laurent.blanc@ec-lyon.fr.
} 
the stochastic approach that has been implemented and its application on a simplified model. In the third and last part, the methodology is validated on an industrial finite element model of a stator vane sector.

\section{Dynamical behaviour of monoblock stator vane sectors}

Figure 1 shows the numerical model which has been used for the simulations and which is located on the first stage of the research compressor CREATE. CREATE (Compresseur de Recherche pour l'Etude des effets Aérodynamiques et TEchnologiques) is a three-stage research compressor which has been specifically designed by Snecma Company for the validation of simulation methods [8]. The complete stator vane is composed by many independent sectors with various numbers of blades. The studied sector, which will be called RD1 hereafter, owns ten blades. In engine working condition, the upper ferrule of the RD1 is clamped in the compressor casing; so this boundary condition has been considered in all the simulations. An eigenmode simulation has been performed on the tuned version of the Fig. 1 model. Figure 2 shows the evolution of the eigenfrequencies against the mode number.

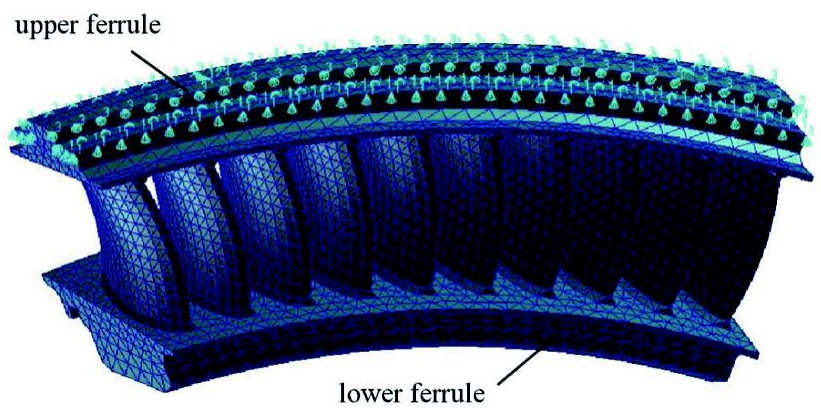

Fig. 1. Numerical model of the studied stator vane sector.

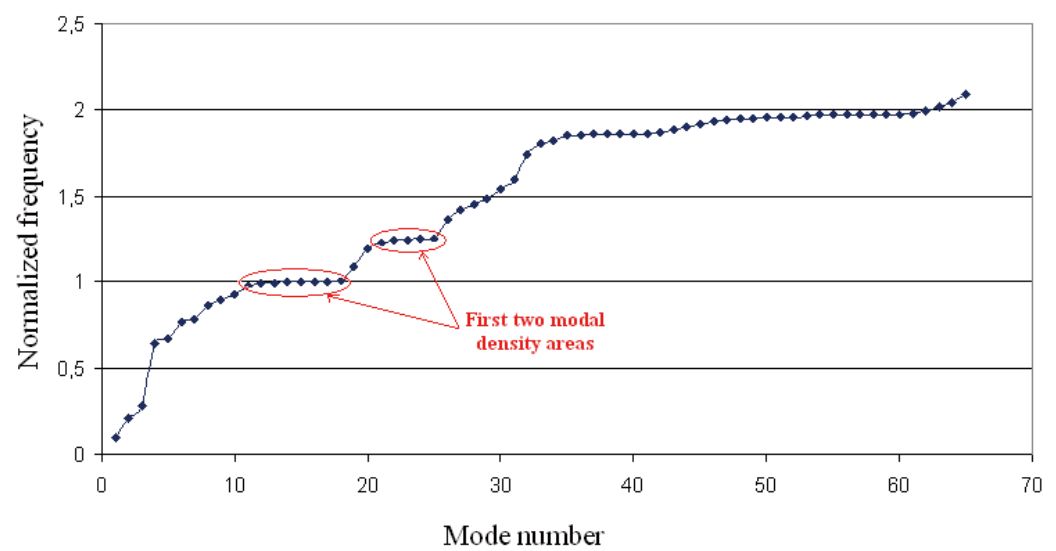

Fig. 2. Diagram representing the eigenfrequencies evolution functions of the eigenmode number (frequencies are normalized with respect to first stage's mean eigenfrequency).

It appears that there are some high modal density areas which are characterized by the presence of stages at different frequency ranges and are mainly composed by the first flexural (1F) and torsional (1T) blade modes (spotted in red on Fig. 2). Two other stages can also be identified, around modes 35 to 42 (approximatively) and around modes 53 to 60: they involve second flexural and torsional blade modes. Inside each stage, modes eigenfrequencies and shapes are really close, which is likely to make the coupling between them more important. Indeed, eigenmode simulations have been performed for different mistuning patterns and the results have been compared to 
those obtained with the tuned model via the MAC criterion [1]. The results which can be found in [10] have shown that the modes located in the high modal density areas are really sensitive to mistuning. The forced response of a stator vane sector is consequently very difficult to predict because the eigenmode shape of some modes changes radically. Therein lies the whole point to rely on a stochastic approach which would allow to determine statistically the most critical areas of a given mistuned sector. Hereafter, industrial example focuses on modelling the first high modal density areas, including modes 10 to 26 .

\section{Projection of the random eigenmodes on a stochastic basis}

Let us consider the deterministic eigenvalue problem for the tuned structure:

$$
\left([K]-\omega_{k}^{2}[M]\right)\left\{\phi_{k}\right\}=0 .
$$

$[K],[M], \omega_{k}$ and $\left\{\phi_{k}\right\}$ respectively designate stiffness and mass matrices, eigenpulsations and eigenvectors. It is assumed that mistuning effects can be summarized by uncertainty on Young's modulus. In order to introduce the randomized problem more easily, and to simplify theoretical developments, the studied stator vane sector is firstly supposed to be made of a single material whose Young's modulus is chosen as the only random parameter; so mass remains deterministic. A random stiffness matrix is defined by adding a random part $[K]$ to the stiffness matrix:

$$
[K]=\xi\left(\frac{\sigma}{m}[K]\right)
$$

where $\xi$ is a reduced random variable, $\sigma$ and $m$ being the given standard deviation and mean value of Young's modulus. The random eigenproblem is:

$$
\left([K]+[K]-\tilde{\omega}_{k}^{2}[M]\right)\left\{\phi_{k}\right\}=0,
$$

where $\tilde{\omega}_{k}$ and $\left\{\phi_{k}\right\}$ are respectively the random eigenpulsations and eigenvectors. The purpose is to assess numerically for the statistical values - mean and standard deviation - of $\tilde{\omega}_{k}$ and $\left\{\phi_{k}\right\}$.

\subsection{Strategy choice}

Such problem can be solved by Monte Carlo simulation: it is the most widespread method, which consists of performing deterministic calculations for a large number of draws of $\xi$ values. Elaborate ways of drawing can reduce the calculation time, but this method requires expensive calculations to get converged statistical descriptors. Monte Carlo simulation is generally used to provide reference results.

Another approach is proposed by Stochastic Finite Element Methods: they consider randomness as an additionnal dimension of a problem defined by a finite element model. Two different ways of dealing with this new dimension can be found in literature. Firstly, perturbation methods are based on a small mistuning hypothesis and lead to Taylor developments of random variables. Successive calculations are then performed from the first order of development to upper ones. Beyond order 2 calculations complexity increases quickly. Moreover, for modal approaches, perturbation methods lead to convergence problems around resonance, unless perturbation is associated with modal quantities directly instead of nodal displacements [7]. Apart from perturbation strategies, the second approach is the Stochastic Spectral Finite Element Method: the idea is to obtain a polynomial expression - depending on $\xi$ - of random unknowns, in association with a Karhunen-Loève description of random parameters [6], this approach is very well suited for the representation of gaussian processes.

The last method, also called "polynomial chaos", is retained here. Indeed, in spite of assuming low levels of mistuning, large variations of modal parameters are expected and sufficiently high orders of random variable development needed (this is confirmed a posteriori). Moreover, no information is available in open literature con- 
cerning the best suited description of randomness for stator vane sectors, then gaussian processes are priviledged (nevertheless, non-gaussian laws could be used through the generalized chaos approach [16]).

It must be mentioned that implemented algorithms are generalized to various numbers of random parameters, when a specific Young's modulus parameter is associated with a specific subset of blades.

Some theoretical details are given below. Then, the method is applied to an Euler-Bernoulli truss model and to an industrial case. Monte Carlo simulations are used to verify polynomial chaos results

\subsection{Polynomial chaos background equations}

The $Q$-dimension and $C$-order polynomial chaos is defined as the whole of the multidimensional Hermite polynomials function of $\left\{\xi_{1}, \ldots, \xi_{Q}\right\}$ and whose degree is inferior or equal to $C$; the $\xi_{i}$ are reduced centered gaussian random variables [6]. Here, only the unidimensional polynomial chaos function of $\xi$ is be presented. The unidimensional Hermite polynomials are obtained by the formula:

$$
\Psi_{n}(\xi)=(-1)^{n} e^{\frac{1}{2} \xi^{2}} \frac{d^{n}}{d \xi^{n}} e^{-\frac{1}{2} \xi^{2}}, n=\{0, \ldots, C\}
$$

It is on this orthogonal polynomials basis that all the random parameters of the system will be decomposed. The random eigenvalues problem is given by Eq. (3). Following [4], the $\tilde{\omega}_{k}^{2}$ are decomposed on the Hermite polynomials basis $\Psi_{n}(\xi)$ :

$$
\tilde{\omega}_{k}^{2}=\omega_{k}^{2} \sum_{n=0}^{N-1} a_{n} \Psi_{n}(\xi)
$$

where $\mathrm{N}$ corresponds to the number of Hermite polynomials which intervene in the decomposition. In the unidimensional case, $\mathrm{N}$ is equal to the polynomial chaos order. The $\left\{\phi_{k}\right\}$ are decomposed on the deterministic modal basis with the assumption that it contains no eigenmode with a multiplicity higher than one:

$$
\left\{\phi_{k}\right\}=\sum_{p=1}^{P} \lambda_{p}\left\{\phi_{p}\right\}
$$

where $P$ represents the number of retained modes from the deterministic modal basis and $\lambda_{p}$ can be projected on the polynomial chaos basis:

$$
\lambda_{p}=\sum_{n=0}^{N-1} \lambda_{p}^{n} \Psi_{n}(\xi)
$$

After the double projection, the random eigenvectors can be rewritten:

$$
\left\{\phi_{k}\right\}=\sum_{p=1}^{P} \sum_{n=0}^{N-1} \lambda_{p}^{n} \Psi_{n}(\xi)\left\{\phi_{p}\right\}
$$

Then, by the means of a Karhunen-Loeve decomposition, the random part $[K]$ of the stiffness matrix is given by:

$$
[K]=\left[K_{1}\right] \Psi_{1}(\xi)=\left[K_{1}\right] \xi
$$

where $\left[K_{1}\right]$ is the part of the $[K]$ matrix where are located the uncertainties.

All the parameters being defined, the random eigenvalues problem to solve can be written as: 


$$
\left(\left([K]+\left[K_{1}\right] \xi\right)-\omega_{k}^{2} \sum_{n=0}^{N-1} a_{n} \Psi_{n}(\xi)[M]\right) \sum_{p=1}^{P} \sum_{n=0}^{N-1} \lambda_{p}^{n} \Psi_{n}(\xi)\left\{\phi_{p}\right\}=0
$$

The coefficients to determine are the $a_{n}$ and the $\lambda_{p}^{n}$. They are calculated via an iterative algorithm resolution such as the Newton-Raphson method.

\subsection{Application}

The simplified model which has been studied is the one presented on the Fig. 3. It is a truss model whose structure looks like a two-blades stator vane sector one. The ferrules which are responsible for the vibratory transfer between the two blades have been defined stiffer than the blades for more realism.

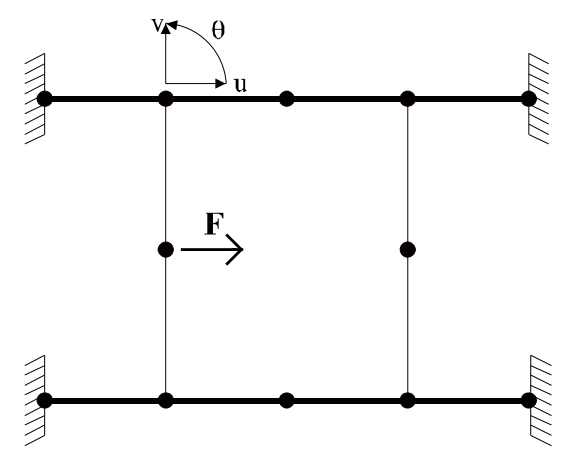

Fig. 3. Studied beam model.

The blades Young's modulii only have been considered as random parameters. Two random variables have been considered so that each blade is independantly mistuned from its neighbor. The stochastic method which has been previoulsy presented for a one variable case has been extended to a two variables application. The random Young's modulus of each blade can be written as:

$$
\tilde{E}_{i}=\bar{E}\left(1+\frac{\sigma_{E}}{\bar{E}} \xi_{i}\right)=\bar{E}\left(1+\delta \xi_{i}\right)
$$

where $\bar{E}$ is the mean Young's modulus, $\sigma_{E}$ is the standard deviation and $\xi_{i}$ is the reduced centered gaussian random variable related to the $i$-th blade.

\subsubsection{Determination of the random eigenmodes}

Figures 4 and 5 show the response surfaces which allow to follow the evolution of the eigenpulsation and the eigenvector of the flexural degree of freedom (dof) of the blade 1 for different values of the two random variables. The interest is to compare the response surfaces obtained by the Monte Carlo simulation and by the polynomial chaos method which has been presented.

5000 draws of couples $\left(\xi_{1}, \xi_{2}\right)$ are performed, following gaussian distributions. This is not sufficient to guarantee convergence for the Monte Carlo method but significant qualitative comparison can be made at reasonnable computational cost. The ranges of parameters on figures are chosen so that couples $\left(\xi_{1}, \xi_{2}\right)$ sweep intervals such that more than $99.99 \%$ of Young's modulii values can be reached.

There is globally a convergence effect that can be observed from the 3rd-order chaos to the 5th-order chaos. Indeed, the convergence seems to be reached in the case of the evolution of the eigenpulsations and also in the case of the evolution of the eigenvectors because the obtained response surfaces show low discrepancies between the Monte Carlo simulation and the polynomial chaos one. So, it is possible, via the presented method, to follow the evolution of a given eigenmode with the mistuning. 
Comparison between $\mathrm{MC}$ and a 3rd-order chaos Standard deviation: $0.5 \%$

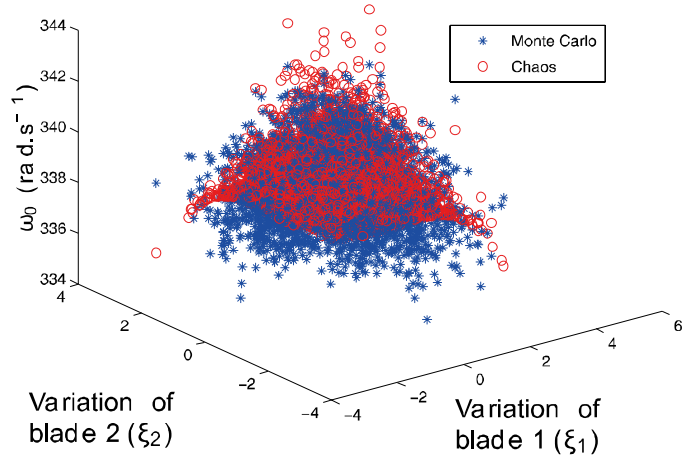

Fig. 4. Response surface of the eigenpulsations - Effect of the polynomial chaos order.

Comparison between MC and a 3rd-order chaos Standard deviation: $0.5 \%$

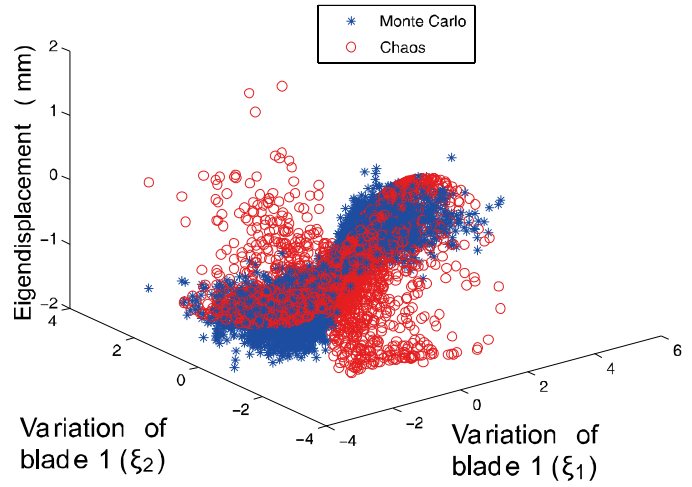

Comparison between MC and a 5th-order chaos Standard deviation: $0.5 \%$

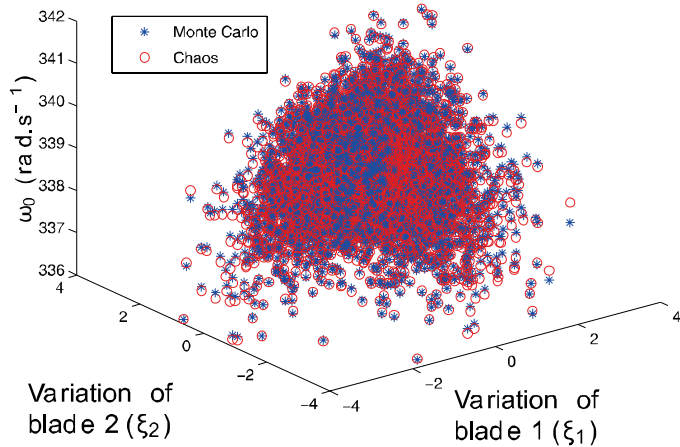

Fig. 5. Response surface of the eigenvectors - Effect of the polynomial chaos order.

\subsubsection{Determination of the first statistic moments of the random forced response}

The calculation of the random eigenmodes allowed to determine the first statistic moments (mean and standard deviation) of the forced response of the model. The displacement is given by:

$$
\{U\}=\sum_{j} \frac{\left\{\Phi_{j}\right\}\left(\left\{\Phi_{j}\right\}^{\mathrm{T}}\{F\}\right)}{-\omega^{2}+\tilde{\omega}_{j}^{2}(1+\mathrm{i} \eta)}
$$

where $j$ is linked to the different modes that were retained while determining the random eigenmodes, $\{F\}$ is the force vector and $\eta$ designates the modal damping. The $\left\{\Phi_{i}\right\}$ random eigenmodes are expanded on the polynomial chaos basis, which enables one to determine the eigenvector associated with a given draw. Then, for a high number of draws and using the Monte Carlo method on Eq. (12), it is possible to calculate the first statistic moments without solving expensive matricial problems. To validate the results, the mean of the displacement obtained by the proposed method is compared to the one obtained by the Monte Carlo method directly applied on the deterministic model, i.e. by solving:

$$
\left((1+\mathrm{i} \eta) \sum_{\mathrm{q}=0}^{\mathrm{Q}}\left[\mathrm{K}_{\mathrm{q}}\right] \Psi_{\mathrm{q}}-\omega^{2}[\mathrm{M}]\right)\{U\}=\{F\}
$$


where $Q$ corresponds to the total number of random variables.

The study has been performed on the model shown on Fig. 3 with the following conditions:

- Unit force applied on the flexural dof of the first blade

- Relative standard deviation $\delta$ is $0.5 \%$

- Modal damping is $0.1 \%$

- 20000 draws

The results on Fig. 6 show the mean value and the standard deviation of the displacement of the dof where the force is applied. Figure 6 shows, on the one hand, the mean of the displacement of the flexural dof of the first blade for a range of frequencies close to the first two eigenfrequencies of the model, and, on the other hand, the standard deviation of the displacement of the same dof. On the graph representing the mean, the deterministic curve reveals two peaks corresponding to the two first eigenmodes of the model, which are pure flexural blade modes. Nevertheless, amplitude varies a lot with the mistuning level, for a given forcing frequency. This explains why the Monte Carlo mean is smooth contrary to the deterministic curve. One remarks that polynomial chaos convergence is reached over the 4th order, then the discrepancy between Monte Carlo and chaos is globally very low. Indeed, the maximum relative gap is about $1.6 \%$ for the 5 th order. The convergence of displacement standard deviation is even better for the same order. Indeed, 1st order discrepancies (when comparing with Monte Carlo) appear around extreme frequencies whereas 5 th order better meets the reference on the whole range of frequencies. The maximum relative discrepancy between 5th order polynomia and Monte Carlo is about $8 \%$. This result is nonetheless satisfactory because the studied configuration is more critical due to the fact that the ferrules have been considered to be much stiffer than the blades. This tends to bring closer the eigenfrequencies of both studied modes. This has been checked, for academic purposes, by reducing artificially the ferrules stiffnesses - and wandering from real configuration: the method converged at the 3 rd order, with a much lower maximum relative discrepancy.
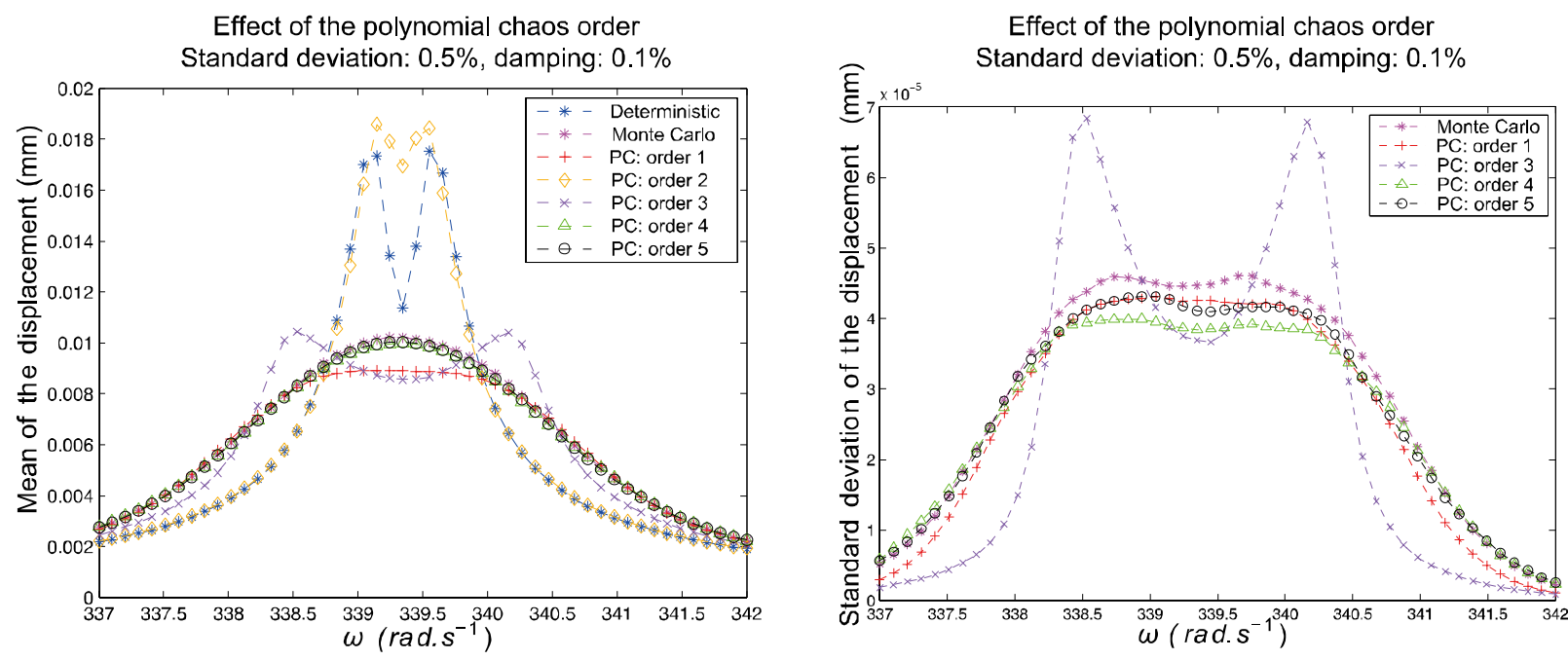

Fig. 6. Forced response: mean of the displacement of the 1st blade flexural dof (left) and relative discrepancy in comparison to Monte Carlo (right) - Effect of the polynomial chaos order.

\section{Industrial application}

From now on, the numerical model is the one shown on Fig. 1, which represents a ten-bladed stator vane sector of the CREATE2-bis test rig. With a view to determining the random eigenmodes of this model by the method presented above, it is necessary to reduce the size of this model. 


\subsection{Model reduction}

The first step is to reduce the numerical model by the Craig-Bampton method [3]. A superelement is designed from a single blade sector. Care is taken to retaining the boundary dofs, used afterwards to put together the different sectors. The convergence of the reduced model is checked by comparison with the reference model. To include uncertainty in this model, the strategy is to associate a single random parameter to the Young's modulus of a given single blade sector, thus this parameter remains in factor of the reduced stiffness matrix as it was in factor of the whole stiffness matrix.

\subsection{Determination of the random eigenmodes}

Using the reduced model, the 17 random eigenmodes of the high modal density areas (modes 10 to 26 on Fig. 2) are expanded on a 3rd order polynomial chaos and on a modal basis made of the first 35 deterministic eigenmodes. Considering the results of the academic example, 3rd order should be unsufficient, nevertheless it is chosen to give a system of reasonable size and reveals to be quite satisfactory as shown below. Also for size reason, only two variables are retained and the studied mistuning pattern is an $\mathrm{ABAB}$ one ( $\xi_{1}$ being the random variable related to $\mathrm{A}$ and $\xi_{2}$ the one related to B). It would obviously be very expensive to consider as many variables as the number of blades.

The random eigendisplacements are then computed on the physical dofs for 20000 draws and the discrepancy criterion Eq. (14) is used to quantify the methods convergence. Let $\left\{\Phi_{m c}\right\}_{k}^{i}$ be the random eigenvector related to the $k$-th random eigenmode and to the $i$-th draw et $\left\{\Phi_{p c}\right\}_{k}^{i}$ be the expansion of this random mode on the polynomial chaos basis. The following discrepancy criterion is defined for the $k$-th mode and for the $i$-th draw:

$$
\operatorname{err}_{k}^{i}=\frac{\left\|\left\{\Phi_{m c}\right\}_{k}^{i}-\left\{\Phi_{p c}\right\}_{k}^{i}\right\|}{\mid\left\{\mid \Phi_{m c}\right\}_{k}^{i} \|}
$$

This criterion gives a global estimation of the discrepancy because, for each mode $k$ and each draw $i$, the discrepancies for all the nodes are taken into account. 20000 draws are performed, then a mean value and a standard deviation of $\operatorname{err}_{k}^{i}$ are computed for each mode (Fig. 7). The error mean value is globally inferior to $1 \%$ and so it is for the standard deviation. This result is very satisfactory because it shows that the chaos is predictive enough regarding the random eigenmodes prediction. Because the forced response can be computed by modal superimposition, a good prediction of the random modes should give good results regarding the random forced response.

Moreover, the calculation-time saving is very significant when using random eigenmodes. Indeed, all the simulations have been performed on the same dual-core processor and the chaos method is 6 times faster than Monte Carlo simulations. The advantage of the method consists in calculating the random eigenmodes once and for all, it is then almost instantaneous to get any response for specific values of the random variables, contrary to the Monte Carlo method for which each draw requires a new expensive deterministic calculation.

\subsection{Determination of the random forced response to a realistic load}

The random eigenmodes are then used as a projection basis to compute the forced response to moving wakes provoked by the upstream rotor blades. The associated unsteady pressure loads are provided by CFD computations performed at nominal engine speed [9]. The sensitivity of each blade to the given mistuning pattern is quantified on Fig. 8. It shows, for each blade, the displacement mean amplitude at mid-span on the trailing edge (indicated by circles) and the amplitude span (indicated by vertical bars) when $\xi_{i}$ varies between -1 and 1 , i.e. when the Young's modulii vary between $-\sigma_{E}$ and $\sigma_{E}$ around their nominal value. The agreement between chaos and Monte Carlo computations is quite satisfactory for a $3 \mathrm{rd}$ order chaos, especially for the highest amplitudes. The chaos results are obtained 30 times faster than the Monte Carlo ones with 20000 draws. A spin-off of these results could be 


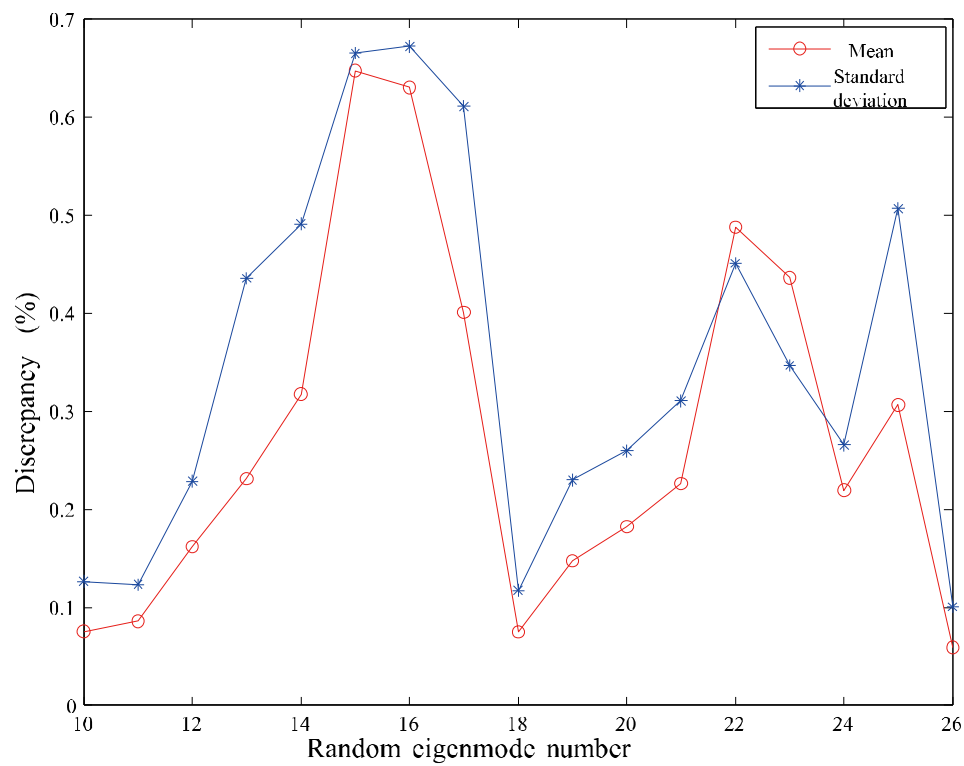

Fig. 7. Mean and standard deviation of the discrepancy between Monte-Carlo and chaos, for each random eigenmode, and for a standard deviation of $1 \%$.

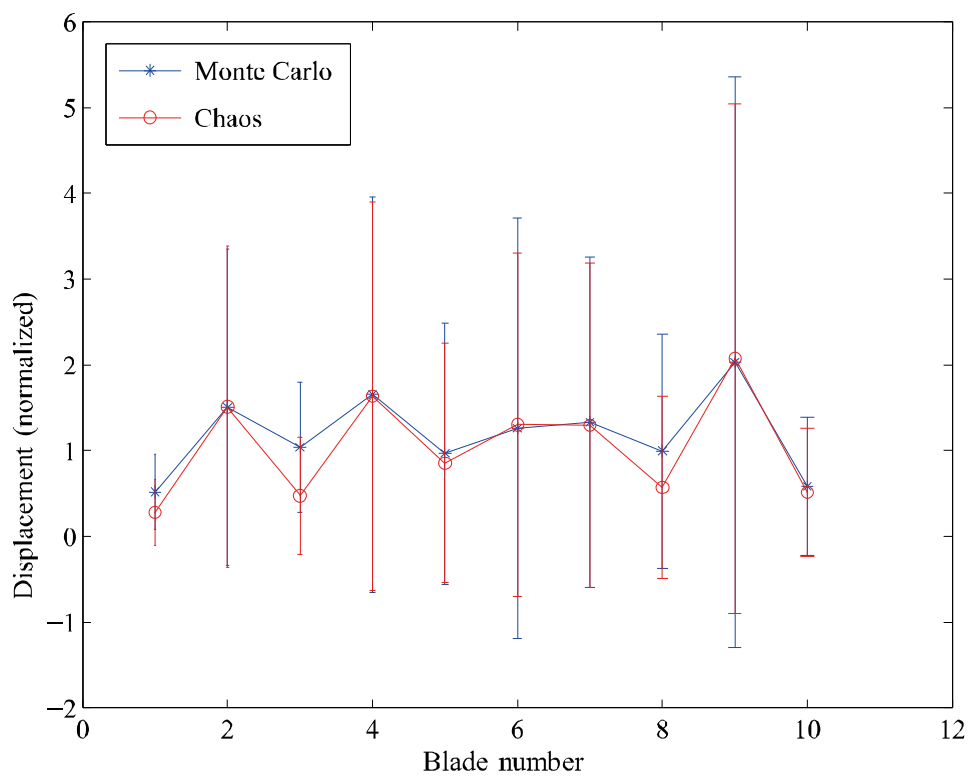

Fig. 8. Displacement at mid-span on the trailing edge. Mean and deviation at $\pm \sigma$ for Monte-Carlo and chaos, for a standard deviation of $1 \%$.

a guideline for prototype test designers: indeed, strain gages should be put first and foremost on the blades prone to high vibratory levels (here blade 9 , then blade $4 . .$. ).

\section{Conclusion}

Problems related to the prediction of the dynamical behaviour of sectored stator vanes have been presented. A stochastic approach has been proposed, expanding eigenmodes on the polynomial chaos basis to compute random 
eigenmodes and then follow the mistuning effects. This approach has been validated on a simplified model with beamlike blades and ferrules. Polynomial chaos and Monte Carlo give very close results. Then, an industrial 3D problem has been adressed. Random eigenmodes and forced responses have been calculated through the proposed method with a significant computation time diminution, offering a promising tool for designers seeking for the most mistuning sensitive areas and the vibratory amplitudes likely to be reached.

The main prospect, concerning the method is to include the uncertainties on the excitation in terms of rotation speed and pressure field. Concerning the industrial example, it is planned to take the aerodynamical damping into account to get more realistic forced computation results. Then these results will be validated by measurements on the CREATE compressor: the experiments have been performed, post-processing is in progress.

\section{Acknowledgments}

Thanks go to Snecma Company for its technical and financial support. This work takes place in the framework of the MAIA mechanical research and technology program sponsored by CNRS, ONERA and SAFRAN Group.

\section{References}

[1] R.J. Allemang, The modal assurance criterion - twenty years of use and abuse, Sound and Vibration Magazine 37(8) (2003), 14-21.

[2] E. Capiez-Lernout and C. Soize, Specifying manufacturing tolerances for a given amplification factor: A nonparametric probabilistic methodology, Number GT2003-38050 in Proceedings of ASME Turbo Expo 2003, 2003.

[3] R.R. Craig and M.C.C. Bampton, Coupling of substructures for dynamic analysis, AIAA Journal 6(7) (1968), $1313-1319$.

[4] O. Dessombz, Analyse Dynamique de Structures comportant des Paramètres Incertains, PhD thesis, École Centrale de Lyon, Lyon, France, December 2000.

[5] R.C.F. Dye and T.A. Henry, Vibration amplitudes of compressor blades resulting from scatter in blade natural frequencies, ASME Journal of Engineering for Power 91(3) (1969), 182-188.

[6] R.G. Ghanem and P.D. Spanos, Stochastic Finite Elements: A Spectral Approach, Springer-Verlag, 1991.

[7] B.R. Mace and P.J. Shorter, A local modal/perturbational method for estimating frequency response statistics of built-up structures with uncertain properties, Journal of Sound and Vibration 242(5) (2001), 793-811.

[8] X. Ottavy, N. Gourdain and A. Vouillarmet, Experimental and numerical investigation of unsteady flows in a high speed three stages compressor, Proceedings of European Turbomachinery Conference, 2009, pp. 247-266.

[9] A.C. Sall, F. Thouverez, L. Blanc, C. Gibert, X. Ottavy, P. Kulisa and P. Jean, Étude du comportement vibratoire des redresseurs sectorisés monobloc: Approches numérique et expérimentale, Proceedings of Congrès Françcais de Mécanique, CFM 2011, Besançon, France, 2011.

[10] A.C. Sall, F. Thouverez, L. Blanc, P. Jean and X. Ottavy, Prediction of the vibratory behaviour of monoblock sectorised stator vanes, Number GT2010-22698 in Proceedings of ASME Turbo Expo 2010, June 2010.

[11] S.H. Shin, S.J. Lee and H.H. Yoo, Statistical approach to analyze vibration localization phenomena in periodic structural systems, Journal of Mechanical Science and Technology 19(7) (2005), 1405-1413.

[12] D.L. Thomas, Dynamics of rotationally periodic structures, International Journal for Numerical Methods in Engineering 14(1) (1979), 81-102.

[13] R. Valid and R. Ohayon, Théorie et calcul statique et dynamique des structures à symétries cycliques, La Recherche Aérospatiale 4 (August 1985), 251-263.

[14] D.S. Whitehead, Effect of mistuning on the vibration of turbo-machine blades induced by wakes, ARCHIVE: Journal of Mechanical Engineering Science 1959-1982 (vols 1-23) 8(1) (1966), 15-21.

[15] F.W. Williams, D. Kennedy, G. Wu and J. Zhou, Exact calculation of natural frequencies of repetitive structures, Structural Engineering and Mechanics 4(5) (1996), 553-568.

[16] D. Xiu and G.E. Karniadakis, The Wiener-Askey polynomial chaos for stochastic differential equations, SIAM J Sci Comput 24(2) (2002), 137-167. 

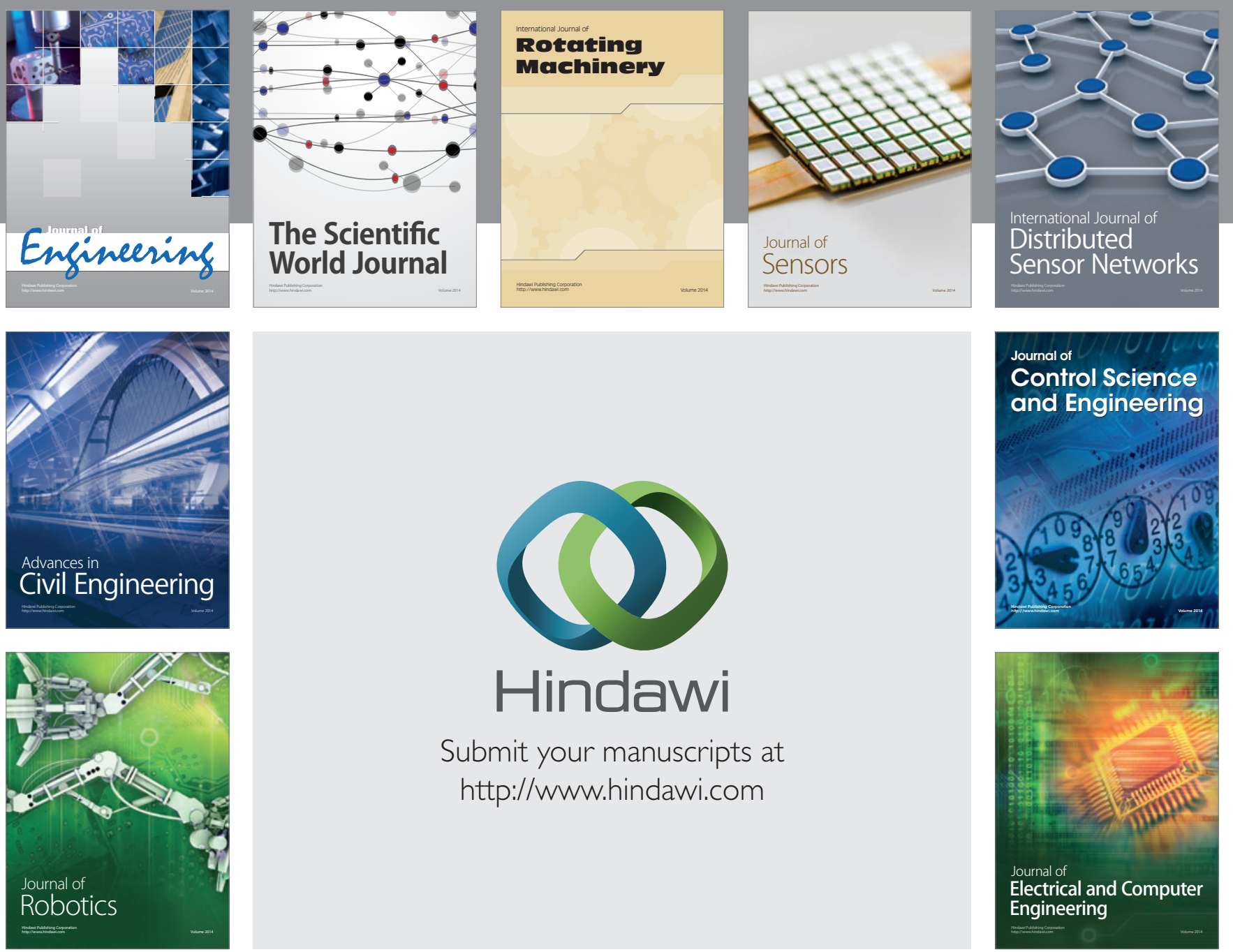

Submit your manuscripts at

http://www.hindawi.com
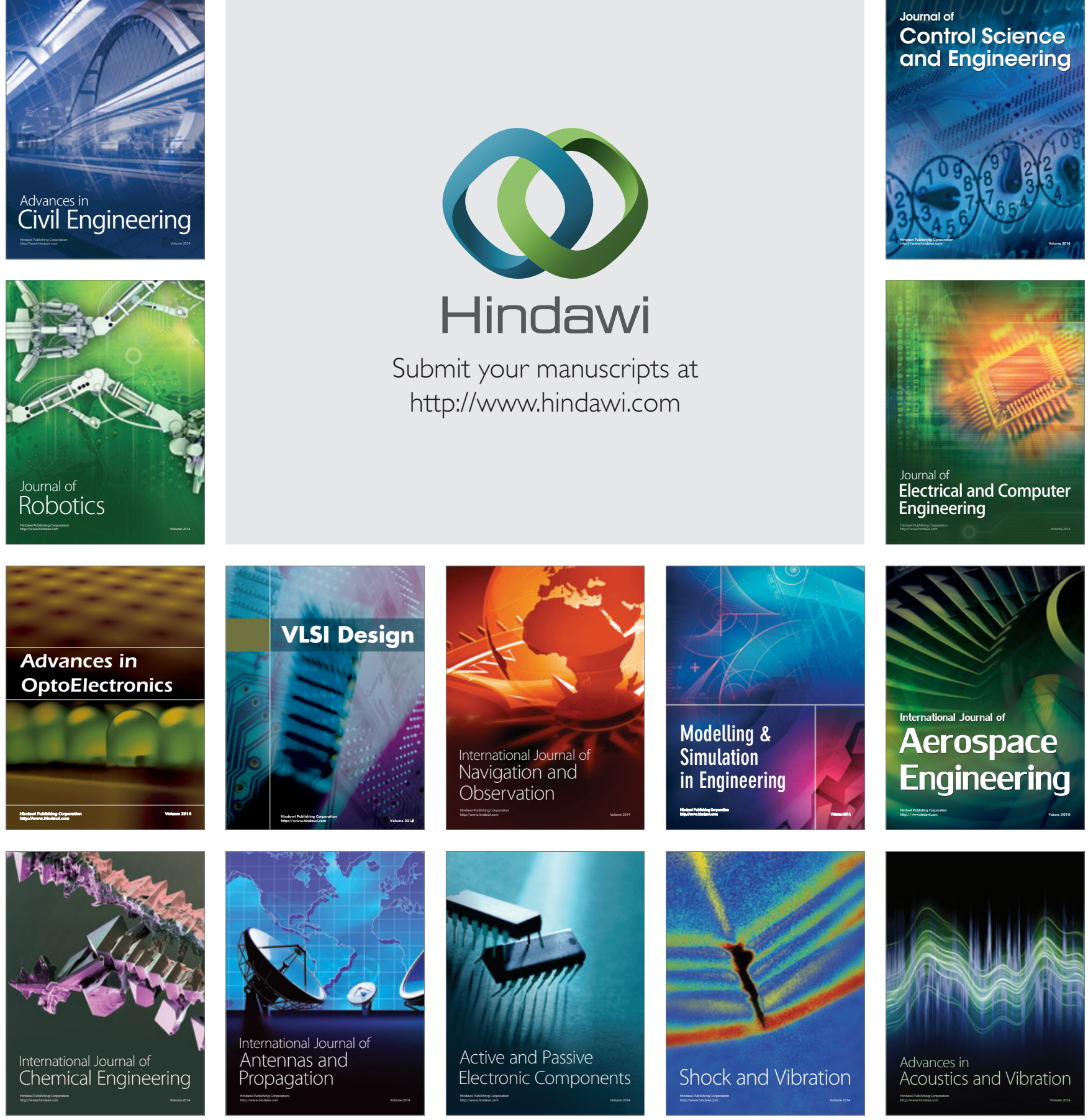